\title{
Immune-related adverse events during anticancer immunotherapy: Pathogenesis and management (Review)
}

\author{
STEFANIA STUCCI, RAFFAELE PALMIROTTA, ANNA PASSARELLI, ERICA SILVESTRIS, \\ ANTONELLA ARGENTIERO, LAURA LANOTTE, SILVANA ACQUAFREDDA, \\ ANNALISA TODISCO and FRANCO SILVESTRIS
}

Department of Internal Medicine and Clinical Oncology, University of Bari Aldo Moro, I-70124 Bari, Italy

Received March 24, 2017; Accepted July 27, 2017

DOI: 10.3892/ol.2017.6919

\begin{abstract}
Immunotherapy is one of the most recent systemic treatments to emerge for use in oncology, and is based on the blocking of inhibitory immune checkpoints to potentiate the immune response to cancer. The anti-cytotoxic $\mathrm{T}$ lymphocyte-associated antigen- 4 antibody ipilimumab and anti-programmed cell death protein 1 antibodies, including nivolumab and pembrolizumab, are currently available and widely used, and other immune-inhibiting antibodies are now under intensive investigation. These antibodies have shown efficacy in a growing number of tumor types, following initial observations of their notable effects in melanoma treatment. Despite the efficacy of these antibodies, their novel mechanisms of action are also associated with a new class of side effects called immune-related adverse events (IRAEs). These side effects do not share a common pathophysiology with other anticancer treatments and, therefore, they often require specific therapies. When detected early and correctly treated, IRAEs are reversible; however, they can become severe and life-threatening if underestimated or inappropriately treated. This review aims to revisit the pathogenesis of IRAEs, with attention to gastrointestinal manifestations, since these are common and potentially dangerous complications of immunotherapy and represent a major cause of treatment discontinuation. Recommendations and guidelines for the management of IRAEs are also presented, in order to provide a clear and applicable algorithm for use by clinicians.
\end{abstract}

Correspondence to: Dr Raffaele Palmirotta, Department of Internal Medicine and Clinical Oncology, University of Bari Aldo Moro, 11 Piazza Giulio Cesare, I-70124 Bari, Italy

E-mail: raffaelepalmirotta@gmail.com

Key words: immunotherapy, immune-related adverse-events, management, cytotoxic $\mathrm{T}$ lymphocyte-associated antigen-4, programmed cell death protein 1 , programmed cell death protein ligand 1

\section{Contents}

1. Introduction

2. Epidemiology of IRAEs

3. Pathogenesis of IRAEs

4. Management of IRAEs

5. Conclusions and future perspectives

\section{Introduction}

Immunotherapy has become a new paradigm in cancer treatment since the reinforcement of immune surveillance has been shown to represent an efficacious approach to cancer immunotherapy (1). In this context, the interactions between tumor cells and stromal cells regulate the release of soluble factors that promote tumor progression, including vascular endothelial factor, interleukin- 6 and transforming growth factor- $\beta$, which promote effector immune cells to strengthen their role in immune surveillance (2).

The inflammatory tumor microenvironment affects cross-talk between $\mathrm{T}$ cells and antigen-presenting cells (APCs), as well as between T cells and tumor cells, resulting in impaired cytotoxicity (3). Therefore, the antitumor response is regulated by the alternative expression of either activating or inhibiting immune checkpoint proteins by immune cells (4). In the context of cancer immunotherapy, two immune checkpoint receptors have been primarily studied, namely cytotoxic T lymphocyte-associated antigen 4 (CTLA4) and programmed cell death protein 1 (PD1), which are inhibitory receptors that control $\mathrm{T}$ cell activation in immune responses at various levels (5).

CTLA4 is exclusively expressed on T cells and primarily regulates the amplitude of the early stages of $\mathrm{T}$ cell activation, acting as a counterbalance to the activity of the co-stimulatory receptor cluster of differentiation (CD) 28 (5). Furthermore, the activation of $\mathrm{T}$ cells requires two sets of signals: The engagement of $\mathrm{T}$ cell receptors (TCRs), and a second signal that results from the binding of co-stimulatory receptors on the T cell with cell-surface molecules on APCs (5). CTLA4 is homologous to $\mathrm{CD} 28$ and the two receptors are localized on the surface of $\mathrm{T}$ cells where they compete to bind to the co-stimulatory ligands CD80 (also termed B7.1) and CD86 (also termed B7.2) on APCs (4-6). Furthermore, CTLA4 
and CD28 are centrally important for the initial activation of naïve $\mathrm{T}$ cells induced by the migration of activated APCs to lymphoid organs (6).

In contrast to CTLA4, the principal inhibitory role mediated by PD1 is to limit the activity of $\mathrm{T}$ cells in peripheral tissues (including tumors) through the inhibition of TCR signaling and the downregulation of anti-apoptotic molecules and pro-inflammatory cytokines. PD1 expression on the surface of $\mathrm{T}$ cells occurs due to chronic and continuous antigen exposure and consequent lymphocyte activation, and defines an anergic or exhausted state. PD1 recognizes two known ligands, programmed death ligand (PD-L)1 (also known as B7-H1) and PD-L2 (also known as B7-DC), which are expressed on tumor cells, APCs and certain non-hematopoietic cells (4). Consequently, PD1/PD-L1 interaction induces apoptosis, inhibits $\mathrm{T}$ lymphocyte proliferation, survival and effector functions, and promotes the differentiation of $\mathrm{T}$ cells into Forkhead box P3-positive regulatory $\mathrm{T}$ cells $(4,5)$.

Targeting these immune checkpoints can reinforce endogenous antitumor activity. The human CTLA4-blocking antibody, ipilimumab, demonstrated an overall survival (OS) benefit for patients with advanced melanoma (7). In addition, two PD1-blocking antibodies, pembrolizumab and nivolumab, have also exhibited favorable clinical activity in melanoma and other solid tumors, including non-small cell lung cancer, renal cell cancer, ovarian cancer and head and neck cancers $(8,9)$.

Despite their clinical efficacy in terms of progression-free survival and OS, these immune checkpoint inhibitors induce novel toxicities in the form of tissue-specific inflammation or immune-related adverse events (IRAEs). Commonly affected tissues include the skin (rash, pruritus and vitiligo), bowel (diarrhea and colitis), liver (hepatitis and elevated liver enzymes) and endocrine glands (hypophysitis, hypothyroidism, thyroiditis and adrenal insufficiency) $(10,11)$. These effects are mediated by $\mathrm{T}$ cell hyperactivation against self-antigens, similarly to autoimmune disorders, and have been more frequently reported with anti-CTLA4 blockade compared with PD1/PD-L1 inhibition (10). Even if responsive to corticosteroids and immunosuppressive agents, such as tumor necrosis factor-blocking antibodies for colitis or mycophenolate mofetil for hepatitis, IRAEs occasionally lead to discontinuation of treatment (11).

New evidence has shown improved clinical responses with the combination of anti-PD1 and anti-CTLA4 antibodies, at the cost of a higher incidence of drug-associated AEs of grade 3 or 4 compared with monotherapies (12). In a previous study, although the majority of IRAEs were treated and controlled, the discontinuation of the clinical trial due to IRAEs in the patient cohort under combination treatment was higher $(55 \%)$ compared with the monotherapy with anti-PD1 or anti-CTLA4 antibodies $(12,13)$.

Considering the promising results of immunotherapy, a coadjutant factor reducing the IRAEs could be advisable to avoid the use of corticosteroids or other immunosuppressive agents. This review revisits the recent advances in the knowledge of IRAEs, focusing on their pathogenesis, to propose novel strategies limiting these effects without interfering with the clinical efficacy of immunotherapy.

\section{Epidemiology of IRAEs}

IRAEs occur in up to $90 \%$ of patients treated with anti-CTLA4 and in $70 \%$ of those receiving anti-PD1/PD-L1 antibodies (Table I) (14,15). Grade 1 and 2 events are most common in the skin and the bowel, whereas grade 3 and 4 toxicities are prevalent in the digestive tract $(16,17)$. The majority of IRAEs occur within 3-6 months of therapy $(16,17)$ and the risk of developing IRAEs appears to be dose-dependent for anti-CTLA4 antibodies (18), but not for anti-PD1 agents. The combination therapy of ipilimumab with nivolumab provides significant clinical results, but also severe toxicities (9). The rate of grade 3 and 4 toxicities for such a combination is $55 \%$, in contrast to monotherapies with nivolumab or ipilimumab, which have frequencies of 16 and $27 \%$, respectively (9). Several studies have also described the additional toxic effects of combinatory immune checkpoint inhibition due to their different mechanisms of action $(12,19,20)$.

Skin toxicity is observed in almost half of patients treated with ipilimumab (44\%); the majority of these cases are of grade 1 and 2 toxicity, whereas severe skin toxicity (grade $3-4$ ) is recorded in $<2 \%$ of patients. Skin toxicities observed with anti-PD1 antibodies include rash (14\%), pruritus (10\%), and occasionally psoriasiform eruptions (21). Vitiligo is the most frequent IRAE for anti-CTLA4 and anti-PD1 therapies in patients with melanoma (21). In addition, there have been several reported cases of Sweet's syndrome or Stevens-Johnson syndrome, as well as toxic epidermal necrosis, pyoderma gangrenosum and cutaneous sarcoidosis during anti-CTLA4 therapy, as well as exacerbation of pre-existing conditions, such as eczema, vitiligo or rosacea and extensive alopecia (22). In $\sim 20 \%$ of patients treated with ipilimumab, a rash has been reported in the form of maculo-papular erythema on the trunk, back or extremities, mostly at grade 1 (22). Dry mouth affects $\sim 5 \%$ of patients receiving immunotherapy (16), and oral candidiasis or Sjögren syndrome may also occur, although these are more frequently reported with PD1-inhibitors (14).

The most common gastrointestinal AEs include diarrhea, vomiting and colitis with abdominal pain or causing intestinal perforation (23). Gastrointestinal IRAEs generally occur after 6-7 weeks of treatment and include mesenteric vessel engorgement, bowel wall thickening and fluid-filled colonic distention; colitis may show a diffuse thickening of the colon wall on positron emission tomography-computed tomography (23). Colitis appears with mucosal erythema and ulcerations simulating Crohn's disease (23). In a meta-analysis including 10 studies and $>2,000$ patients treated with immune checkpoint inhibitors, diarrhea was frequently reported (11-51\% of cases), whereas vomiting was only described in seven studies (3-32\% of cases) and colitis in six studies (1-16\% of cases) (19). The KEYNOTE-006 randomized open-label study has provided additional insight into the risks of different gastrointestinal toxicities from pembrolizumab (anti-PD1) vs. ipilimumab (anti-CTLA4). The findings revealed that diarrhea is more common during anti-CTLA4 (30\% any grade and $\sim 10 \%$ grade $3-4$ ) than during anti-PD1/PD-L1 therapy. Furthermore, in this trial, the risk of colitis was $8.2 \%$ for ipilimumab vs. $3.6 \%$ for pembrolizumab (21). 
Table I. Incidence of IRAEs during anticancer immunotherapy with anti-CTLA4 or anti-PD1/PD-L1 antibodies.

\begin{tabular}{|c|c|c|}
\hline \multirow[b]{2}{*}{ IRAEs } & \multicolumn{2}{|c|}{ IRAE incidence (all grades), $\%$} \\
\hline & $\begin{array}{l}\text { Anti-CTLA4 } \\
\text { immunotherapy }\end{array}$ & $\begin{array}{l}\text { Anti-PD1/PD-L1 } \\
\text { immunotherapy }\end{array}$ \\
\hline $\begin{array}{l}\text { Dermatological (rash, pruritus, psoriasiform eruptions, vitiligo, Sweet's } \\
\text { syndrome, Stevens-Johnson syndrome, toxic epidermal necrosis, pyoderma } \\
\text { gangrenosum, cutaneous sarcoidosis) }\end{array}$ & 44.0 & 37.4 \\
\hline Gastrointestinal (diarrhea, colitis, hepatitis, pancreatitis) & 30.0 & 20.0 \\
\hline Fatigue & 46.0 & 47.0 \\
\hline Endocrine (thyroid dysfunction, hypophysitis, adrenal insufficiency) & 10.0 & $<10.0$ \\
\hline Musculoskeletal & 6.1 & 7.6 \\
\hline Mucosal toxicity (oral mucositis, dry mouth) & $<5.0$ & 5.0 \\
\hline Respiratory (pulmonitis) & 1.0 & $<1.0$ \\
\hline Ophthalmological (episcleritis, conjunctivitis, uveitis, orbital inflammation) & $<1.0$ & - \\
\hline $\begin{array}{l}\text { Neurological (paresthesia, Guillain-Barré syndrome, aseptic or lymphocytic } \\
\text { meningitis, posterior reversible encephalopathy syndrome, inflammatory } \\
\text { enteric neuropathy, transverse myelitis) }\end{array}$ & $<1.0$ & - \\
\hline Renal (renal failure) & $<1.0$ & $1.0-22.0$ \\
\hline $\begin{array}{l}\text { Hematological (red cell aplasia, autoimmune neutropenia or pancytopenia, } \\
\text { acquired hemophilia) }\end{array}$ & $<1.0$ & - \\
\hline
\end{tabular}

IRAE, immune-related adverse event; CTLA4, cytotoxic T lymphocyte antigen 4; PD1, programmed cell death protein 1; PD-L1, programmed death ligand 1.

Other research has suggested an increased risk of diarrhea and colitis with ipilimumab in comparison to nivolumab treatment. In a recently reported phase 3 trial evaluating combined nivolumab/ipilimumab therapy and monotherapy with ipilimumab or nivolumab, the risk of diarrhea and colitis was observed to be markedly higher in the ipilimumab-containing arms compared with the nivolumab monotherapy arm (12).

In patients treated with ipilimumab for melanoma, liver toxicity has been reported in $\sim 5 \%$ of patients, particularly after 6 weeks of treatment, as elevated levels of hepatic enzymes and bilirubin, or as acute hepatitis (24). Immune-associated elevation of pancreatic enzyme levels has also been reported in immunotherapy-based protocols (25). Checkpoint inhibitor therapy can also induce a spectrum of rare cardiac side effects, including fibrosis, autoimmune myocarditis, cardiomyopathy, heart failure and cardiac arrest (26).

Approximately $5-10 \%$ of patients receiving immune checkpoint inhibitors develop an endocrine IRAE of any grade, including thyroid dysfunction (most often hypothyroidism) (21), hypophysitis (mainly observed with ipilimumab; $10 \%$ ) (27), and adrenal insufficiency (with an incidence of $\sim 6 \%$ for all grades and 1\% for grades 3-4 with nivolumab and pembrolizumab treatment) (28). Fatigue is one of the most common side effects of immune checkpoint blockade, with a frequency of $47 \%$ in patients treated with anti-PD1 agents (16). Immune-associated pneumonitis occurs in $\sim 1 \%$ of patients (29); severe forms are extremely rare with ipilimumab, whereas they have been frequently reported with anti-PD1 agents (30).
Ophthalmological and neurological IRAEs occur in patients treated with ipilimumab (31). Ocular toxicity (incidence, $<1 \%$ ) includes episcleritis, conjunctivitis, uveitis and orbital inflammation (31). Autoimmune neuropathies are rare (incidence, $<1 \%$ ) and include mild paresthesia as well as severe neurological syndromes such as Guillain-Barré syndrome, aseptic or lymphocytic meningitis, posterior reversible encephalopathy syndrome, inflammatory enteric neuropathy or transverse myelitis (32).

Arthralgia and arthritis are the most common rheumatic and musculoskeletal IRAEs, with an incidence of $~ 5 \%$ (33). There have been few reports of sicca syndrome, myositis or severe salivary hypofunction (33). A small number of cases of systemic lupus erythematous or polymyalgia rheumatic and giant cell arteritis have been described with CTLA4 blockade (34). Furthermore, the incidence of arthralgia was higher with combined ipilimumab/nivolumab immunotherapy (10.5\%) compared with ipilimumab or nivolumab monotherapy (6.1 and $7.7 \%$, respectively) in a previous study (33).

Kidney damage, including renal failure in patients treated with anti-CTLA4, has also been reported, with an incidence of $1 \%$ (35); this includes interstitial nephritis, granulomatous nephritis and glomerular lupus-like nephropathy (34). Renal dysfunctions or increases in serum creatinine are more common with nivolumab therapy compared with ipilimumab treatment (incidence, 1-22\%) (36).

Hematological toxicity, including red cell aplasia, autoimmune neutropenia or pancytopenia and acquired hemophilia A, has also been reported in small number of patients receiving anti-CTLA4 antibodies (37). 
A

LYMPH NODE

Priming phase
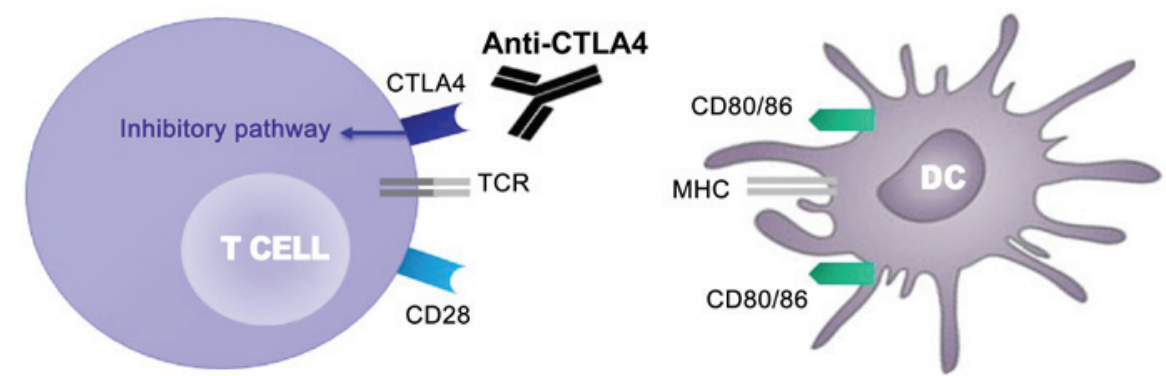

B

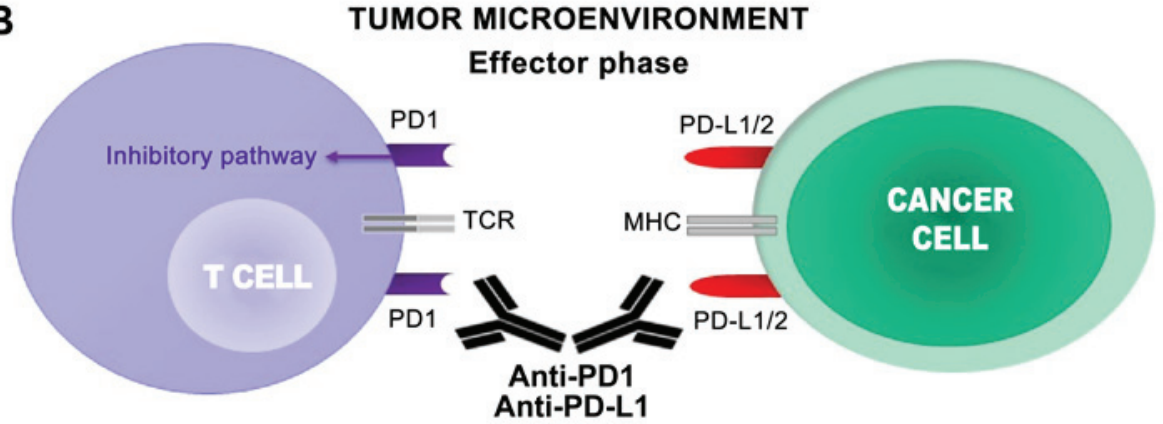

Figure 1. CTLA4 and PD1 regulate different stages of T cell response. (A) T cell activation requires two complementary signals: The interaction between the TCR and peptide-MHC complex must be associated with a second co-stimulatory signal mediated by CD28. Conversely, the binding of CTLA4 to CD80/86 provides a control signal that suppresses ongoing T cell activation. (B) PD1 is upregulated on T cells following persistent antigen exposure. When PD1 binds to its ligand, PD-L1 or PD-L2, expressed by tumor cells, the T cell receives an inhibitory signal. Antibodies against CTLA4 or PD1/PD-L1 can activate T cells. CTLA4, cytotoxic T lymphocyte antigen 4; PD1, programmed cell death protein 1; TCR, T cell receptor; MHC, major histocompatibility complex; CD, cluster of differentiation; PD-L1, programmed death ligand 1; PD-L2, programmed death ligand 2; DC, dendritic cell.

\section{Pathogenesis of IRAEs}

The major immune checkpoint receptors, which include CTLA4 and PD1, perform a pivotal role in regulating the mechanisms of tolerance to self-antigens through the downregulation as well as the prevention of abnormal activity against self-antigens (38) (Fig. 1).

The functions of the CTLA4 and PD1 pathways include the downregulation of $\mathrm{T}$ cell activation, which serves a significant function in the interactions between the immune system and cancer, as this may be attenuated by the influence of tumor cells. Furthermore, the continuous release of antigens by tumor cells within the tumor microenvironment has been shown to upregulate the inhibitory immune pathways as a result of chronic stimulation (4). Infiltrating $\mathrm{T}$ cells are frequently reduced due to the presence of CTLA4 and PD1 within the microenvironment, leading to impaired antitumor immunity (6). Once CTLA4 and PD1 bind to their ligands (CD80/86 and PD-L1/PD-L2, respectively), they negatively regulate intercellular interactions, even in the presence of tumor antigens (4). By blocking these interactions with CTLA4 and PD1, checkpoint inhibitors lead to increased $\mathrm{T}$ cell proliferation and activity, followed by an antitumor response and potentially by autoimmune reactions (39). Notably, certain polymorphisms of these immune receptor genes have been associated with increased susceptibility to various autoimmune diseases $(39,40)$. However, the specific pathogenic mechanisms of IRAEs, which may occasionally be severe and life-threatening, remain largely unknown.
IRAEs have been associated with massive infiltration of highly-activated $\mathrm{CD}^{+}$and $\mathrm{CD} 8^{+} \mathrm{T}$ cells and an increased serum release of inflammatory cytokines (41). The most extensive data on the pathogenic mechanisms of IRAEs derive from studies on immunotherapy with CTLA4 inhibitors, in particular regarding the pathogenesis of anti-CTL4-associated gastrointestinal and dermatological effects. Berman et al (42) demonstrated that anti-CTLA4 agents induced the dysregulation of gastrointestinal mucosal immunity, as highlighted by the perturbation of enteric flora homeostasis. Variations of perinuclear anti-neutrophil cytoplasmic antibody staining and $O m p C(E . c o l i)$ levels, as well as increased levels of neutrophil-derived fecal calprotectin, have been described (42). Despite this, calprotectin was not considered a predictive biomarker of IRAEs, but it only occurs in inflammatory bowel disease, indicating a different pathogenesis (42).

Since CTLA 4 is highly expressed on the surface of regulatory $\mathrm{T}$ cells (Tregs), which have been indicated to downregulate cell-mediated immunity, an alternative hypothesis proposed that anti-CTLA4 antibodies cause an imbalance between Treg activity and effector $\mathrm{T}$ cell function. Indeed, anti-CTLA4 agents can induce significant peripheral blood Treg depletion, despite a high frequency of T-helper (Th)1 and Th17 cell subsets and increased levels of cytotoxic granzyme CD8 ${ }^{+} \mathrm{T}$ cells (43). Conversely, no differences in mucosal FoxP $3^{+}$Tregs in the colonic mucosa were observed between anti-CTLA4-treated patients with and without gastrointestinal IRAEs (44). Furthermore, an expansion of Th17 cells and elevated levels 
Table II. Schematic treatment algorithm for management of gastrointestinal adverse events during anticancer immunotherapy.

A, Diarrhea and colitis.

\begin{tabular}{ll}
\hline Severity of symptoms $\quad$ Description & Management and follow-up \\
\hline
\end{tabular}

Grade 1

Diarrhea: <4 stools/day over baseline.

Colitis: Asymptomatic

Grade 2

Diarrhea: 4-6 stools/day over baseline.

Colitis: Abdominal pain; blood in stool.

Grade 3

Diarrhea: $\geq 7$ stools/day over baseline; incontinence. Colitis: Severe abdominal pain, medical intervention indicated, peritoneal signs.

\section{Continue ICPI}

Supportive care: Oral fluid and anti-motility agents such as loperamide

If symptoms persist: Budesonide $9 \mathrm{mg} /$ daily

Delay ICPI and treat as grade 1

If symptoms persist >5-7 days: 0.5-1.0 mg/kg/day

methylprednisolone or PO equivalent

If no improvement occurs manage as for grade 3-4

Permanently discontinue ICPI

$1.0-2.0 \mathrm{mg} / \mathrm{kg} /$ day methylprednisolone i.v. or equivalent

Consider lower gastrointestinal endoscopy

If symptoms persist: Infliximab $5 \mathrm{mg} / \mathrm{kg}$ every 2 weeks

(if no contraindications)

If symptoms persist: Consider alternative

immunosuppressive therapy (such as mycophenolate mofetil and tacrolimus)

Grade 4 Life-threatening, perforation
As for grade 3

B, Hepatotoxicity.

\begin{tabular}{|c|c|c|}
\hline Severity of symptoms & Description & Management and follow-up \\
\hline Grade 1 & $\begin{array}{l}\text { AST or ALT }>1 \text { to } 3 x \mathrm{ULN} \text {; and/or } \\
\text { total bilirubin } 1.0-1.5 \mathrm{x} \text { ULN }\end{array}$ & $\begin{array}{l}\text { Continue ICPI } \\
\text { Exclude liver injury induced by malignancies, alcohol, } \\
\text { viral hepatitis or drugs }\end{array}$ \\
\hline Grade 2 & $\begin{array}{l}\mathrm{AST} \text { or } \mathrm{ALT}>3.0 \text { to } 5.0 \mathrm{x} \mathrm{ULN} \text {; and/or } \\
\text { total bilirubin }>1.5 \text { to } 3.0 \mathrm{x} \mathrm{ULN}\end{array}$ & $\begin{array}{l}\text { Delay ICPI } \\
\text { If symptoms persist }>5-7 \text { days or worsens: } \\
0.5-1 \mathrm{mg} / \mathrm{kg} / \text { day methylprednisolone i.v. or PO equivalent }\end{array}$ \\
\hline Grade 3-4 & $\begin{array}{l}\text { AST or ALT }>5.0 x \mathrm{ULN} \text {; and/or } \\
\text { total bilirubin }>3.0 \mathrm{x} \text { ULN }\end{array}$ & $\begin{array}{l}\text { Delay or discontinue ICPI } \\
1-2 \mathrm{mg} / \mathrm{kg} / \text { day methylprednisolone i.v. or equivalent } \\
\text { If symptoms persist: Consider alternative } \\
\text { immunosuppression (e.g., mycophenolate mofetil) }\end{array}$ \\
\hline
\end{tabular}

ICPI, immune checkpoint inhibitors; ULN, upper limit of normal; i.v., intravenous; AST, aspartate aminotransferase; ALT, alanine aminotransferase.

of serum IL-17 were associated with gastrointestinal disorders during immunotherapy (45).

Based on these data, a trial of neoadjuvant therapy with ipilimumab at $10 \mathrm{mg} / \mathrm{kg}$ demonstrated that baseline pretreatment with IL-17 is significantly associated with the risk of subsequent development of severe immune-mediated diarrhea (10). Host factors, such as genetic predisposition, associated with IRAEs have not been completely elucidated, although it is plausible that the incidence of gastrointestinal IRAEs may be associated with CTLA4 polymorphism alleles (42).

Dermatological IRAEs, including vitiligo, depend on $\mathrm{T}$ cell activation against melanocytes, which shows a perivascular infiltrate of lymphocytes and eosinophils into the epidermis (10), as well as of Melan-A-specific CD8 ${ }^{+} \mathrm{T}$ cells into the dermis $(46,47)$. Notably, the prophylactic use of immunosuppressive therapy, such as corticosteroids, has not been shown to prevent the incidence of IRAEs (48).

\section{Management of IRAEs}

Gastrointestinal effects. Diarrhea and colitis are common IRAEs associated with anti-CTLA4 or anti-PD-1/PD-L1 use. Management of grade 1 diarrhea (defined as $<4$ stool evacuations above baseline per day) is based on fluid hydration, electrolyte repletion and anti-motility agents such as loperamide (Table II) (49). The cornerstone of diagnosis in patients suspected of severe acute infectious diarrhea (such as Clostridium difficile) is the microbiological analysis of stools, 
Table III. Schematic treatment algorithm for management of dermatological adverse events during anticancer immunotherapy.

Severity of symptoms $\quad$ Description $\quad$ Management and follow-up

Grade 1

Grade 2

Maculopapular rash $<10 \%$ BSA, with/without symptoms (pruritus, burning, tightness)

Maculopapular rash 10-30\% BSA, with/without symptoms (pruritus, burning, tightness); limiting instrumental ADL

Grade 3-4

Maculopapular rash $>30 \%$ BSA, with/without symptoms (pruritus, burning, tightness); limiting self-care ADL; local or extensive superinfection

\section{Continue ICPI}

Supportive care: Anti-histamines and topical steroid Delay ICPI

Topical steroids

If symptoms persist $>7$ days: Systemic steroids (such as methylprednisolone $0.5-1 \mathrm{mg} / \mathrm{kg} /$ day or PO equivalent)

Delay or discontinue ICPI

Methylprednisolone 1-2 mg/kg/day or

PO equivalent

Consider skin biopsy

If symptoms persist: Consider alternative

immunosuppressive therapy (such as

mycophenolate mofetil or infliximab)

ICPI, immune checkpoint inhibitors; BSA, body surface area; ADL, activities of daily living; PO, per os.

in association with a complete blood count and the measurement of serum inflammatory parameters (50). If symptoms persist, $9 \mathrm{mg}$ of budesonide can be administered daily. The prophylactic use of budesonide has been tested during treatment with ipilimumab, without resulting in any difference in the incidence of diarrhea (51). Grade 2 colitis (4-6 episodes of diarrhea per day) can represent a risk factor for the development of a bowel perforation. Persistent symptoms in the absence of perforation require systemic corticosteroid treatment (methylprednisolone $0.5-1 \mathrm{mg} / \mathrm{kg} /$ day or oral equivalent). For severe (grade 3 or 4 ) toxicity, defined as $\geq 7$ stools above baseline per day, peritoneal signs, bowel perforation or fever, immunotherapy should be permanently discontinued. Patients should be hospitalized for clinical monitoring and begin an appropriate intravenous (i.v.) electrolyte repletion. Following endoscopic evaluation, systemic corticosteroid treatment (methylprednisolone 1-2 $\mathrm{mg} / \mathrm{kg}$ i.v. daily or equivalent) should be administered (50). High-dose methylprednisolone is indicated in clinically unstable patients (17). For severe or steroid-refractory symptoms, infliximab administration at a dose of $5 \mathrm{mg} / \mathrm{kg}$ every 2 weeks should be considered. Infliximab is contraindicated in patients with sepsis or intestinal perforation. In steroid- and infliximab-refractory patients, the use of tacrolimus or mycophenolate mofetil may be evaluated (52). However, the development of gastrointestinal toxicities during the administration of one immune checkpoint inhibitor does not preclude the use of another one (17).

Hepatotoxicity. Autoimmune hepatotoxicity manifests as an asymptomatic increase in serum transaminases and total bilirubin. Other causes of liver injury, including alcohol abuse, viral hepatitis or hepatotoxic medications, should be excluded. Ordinarily, the average time to resolution is 8 weeks. For grade 2 toxicity, immunotherapy should be withheld and, in case of persistence, corticosteroids (methylprednisolone $0.5-1 \mathrm{mg} / \mathrm{kg}$ i.v. or oral equivalent daily) are recommended.
For grade 3-4 toxicity, immunotherapy should be permanently discontinued (50). Patients should be hospitalized and receive methylprednisolone (1-2 $\mathrm{mg} / \mathrm{kg}$ i.v.). If symptoms persist, administration of mycophenolate mofetil (50) can be considered, and infliximab is not recommended (53) (Table II).

Dermatological disorders. Dermatological toxicities, including rash, pruritus and vitiligo, often occur in patients treated with immune-checkpoint inhibitors $(54,55)$. During immunotherapy administration, grade 1 rash and pruritus can be managed with topical corticosteroids and systemic antihistamines. Delaying of immunotherapy is mandatory in cases of grade 2 toxicity; methylprednisolone at a dose of $0.5-1 \mathrm{mg} / \mathrm{kg}$ daily or oral equivalent is required with persisting symptoms. Furthermore, grade 3 rash necessitates systemic corticosteroid therapy (methylprednisolone $1-2 \mathrm{mg} / \mathrm{kg}$ daily) and discontinuation of immune-checkpoint inhibitor administration (49). Infliximab or mycophenolate mofetil can be used to manage refractory symptoms (53) (Table III). No treatment for vitiligo has been validated thus far (50).

Pneumonitis. Pneumonitis occurs later than other IRAEs (56). Although rare, it may be fatal, and an effective workup is therefore required. In a patient with a chronic productive cough, shortness of breath and hypoxia, chest X-ray and CT scans are diagnostic. Suspicious signs on CT scan include evidence of consolidative or 'ground glass' opacities with peripheral distribution (57). In mild cases, systemic corticosteroid treatment with prednisone $1-2 \mathrm{mg} / \mathrm{kg}$ or methylprednisolone $1 \mathrm{mg} / \mathrm{kg}$ daily can be administered. In more severe toxicities, hospitalization, discontinuation of the immune-checkpoint inhibitor and high doses of corticosteroids (methylprednisolone $2-4 \mathrm{mg} / \mathrm{kg}$ daily) are indicated, once pulmonary infections have been excluded. If symptoms persist, mycophenolate mofetil, cyclophosphamide or infliximab may also be administered $(53,58)$ (Table IV). 
Table IV. Schematic treatment algorithm for management of pneumonitis, endocrinopathy and renal injury occurring as adverse events during anticancer immunotherapy.

A, Pneumonitis.

\begin{tabular}{|c|c|c|}
\hline Severity of symptoms & Description & Management and follow-up \\
\hline Grade 1 & Asymptomatic; radiographic changes only & $\begin{array}{l}\text { Continue ICPI } \\
\text { Clinical or diagnostic observation }\end{array}$ \\
\hline Grade 2 & $\begin{array}{l}\text { Symptomatic (mild to moderate new } \\
\text { symptoms) }\end{array}$ & $\begin{array}{l}\text { Delay ICPI } \\
1 \mathrm{mg} / \mathrm{kg} / \text { day methylprednisolone or PO equivalent } \\
\text { Consider bronchoscopy and lung biopsy }\end{array}$ \\
\hline Grade 3-4 & $\begin{array}{l}\text { Severe symptoms; worsening hypoxia; } \\
\text { life-threatening }\end{array}$ & $\begin{array}{l}\text { Discontinue ICPI } \\
2-4 \mathrm{mg} / \mathrm{kg} / \text { day methylprednisolone or i.v. equivalent } \\
\text { Consider bronchoscopy and lung biopsy } \\
\text { If symptoms are not improving within } 48 \mathrm{~h} \text { or are } \\
\text { worsening: Consider alternative immunosuppressive } \\
\text { therapy (such as mycophenolate mofetil, } \\
\text { cyclophosphamide or infliximab) }\end{array}$ \\
\hline
\end{tabular}

B, Endocrinopathy.

\begin{tabular}{|c|c|c|}
\hline Severity of symptoms & Description & Management and follow-up \\
\hline Grade 1 & Asymptomatic & $\begin{array}{l}\text { Continue ICPI } \\
\text { Hormone replacement }\end{array}$ \\
\hline Grade 2 & Symptomatic endocrinopathy & $\begin{array}{l}\text { Delay ICPI } \\
1-2 \mathrm{mg} / \mathrm{kg} / \text { day methylprednisolone i.v. or PO equivalent }\end{array}$ \\
\hline Grade 3-4 & $\begin{array}{l}\text { Symptomatic endocrinopathy requiring } \\
\text { urgent medical intervention, interfering } \\
\text { with ADL. Grade 4: Life-threatening } \\
\text { consequences (such as adrenal crisis) }\end{array}$ & $\begin{array}{l}\text { Delay or discontinue ICPI } \\
2 \mathrm{mg} / \mathrm{kg} / \text { day methylprednisolone i.v. or equivalent } \\
\text { If suspicion of adrenal crisis: stress dose of steroids } \\
\text { with mineralocorticoid activity }\end{array}$ \\
\hline
\end{tabular}

C, Renal injury.

\begin{tabular}{|c|c|c|}
\hline Severity of symptoms & Description & Management and follow-up \\
\hline Grade 1 & Creatinine $1.5 x$ ULN & $\begin{array}{l}\text { Continue ICPI } \\
\text { Creatinine monitoring }\end{array}$ \\
\hline Grade $2-3$ & Creatinine $>1.5$ to $6 x$ ULN & $\begin{array}{l}\text { Delay ICPI } \\
0.5-1 \mathrm{mg} / \mathrm{kg} / \text { day methylprednisolone i.v. or equivalent }\end{array}$ \\
\hline Grade 4 & Creatinine $>6 \mathrm{x}$ ULN & $\begin{array}{l}\text { Discontinue ICPI } \\
1-2 \mathrm{mg} / \mathrm{kg} / \text { day methylprednisolone i.v. or equivalent }\end{array}$ \\
\hline
\end{tabular}

ICPI, immune checkpoint inhibitors; ADL, activities of daily living; ULN, upper limit of normal; PO, per os; i.v., intravenous.

Endocrinopathy. Hypothyroidism and hypophysitis are the most common endocrine IRAEs. Therefore, thyroid function must be regularly assessed, whereas the pituitary axis should be tested only in suspected cases (52). Hypophysitis presents with signs of hypopituitarism (fatigue, hypoglycemia, hypotension and hypogonadism) and magnetic resonance imaging shows enhancement and enlargement of the pituitary gland (59). This condition is usually irreversible and requires permanent hormone replacement. When symptoms occur, methylprednisolone
(1-2 mg/kg/day i.v. or oral equivalent) should be administered. A stress dose of i.v. corticosteroids with mineralocorticoid activity is indicated in cases of adrenal insufficiency. Immune checkpoint therapy must be delayed in symptomatic endocrinopathy and permanently discontinued in cases of severe toxicity $(60,61)$. Symptomatic hyperthyroidism is managed with $\beta$-blockers and steroids (62). Once hypothyroidism is documented, hormone replacement represents the treatment of choice, with no indications for the deferral of immunotherapy (Table IV) (11). 
Renal injury. Kidney failure presents with interstitial, granulomatous nephritis or glomerular lupus-like nephropathy. Grade 1 toxicity requires constant monitoring of serum creatinine once a week without discontinuing the ongoing immunotherapy. Interruption of the treatment is recommended in cases of toxicity of grade 2-3 unresponsive to steroids (methylprednisolone $0.5 \mathrm{mg} / \mathrm{kg}$ daily i.v.). In grade 4 toxicity, permanent discontinuation of therapy is mandatory, while renal biopsy and high-dose steroids (methylprednisolone $1-2 \mathrm{mg} / \mathrm{kg}$ daily i.v.) may be useful in cases of kidney impairment (Table IV) (50).

Immunosuppressant effect on anticancer immunotherapy. There is limited knowledge available regarding the impact of corticosteroids on the outcome of cancer treatment. For this purpose, a phase II trial was performed with ipilimumab monotherapy in advanced melanoma. In this study, 83 patients were monitored to determine the disease control efficacy in the presence (52\% of patients) or absence ( $48 \%$ of patients) of steroid treatment for IRAEs (63). Systemic corticosteroid treatment of IRAEs did not appear to impact the development or maintenance of ipilimumab clinical activity in advanced melanoma. By contrast, data are still missing regarding the effects of immunosuppressive agents for IRAEs on the outcome of anti-PD1 therapy (64). Therefore, additional studies are required to understand whether the management of IRAEs with immunosuppressive agents has a detrimental effect on antitumor immunity.

\section{Conclusions and future perspectives}

Despite the promising results of trials with immunotherapeutic agents, this type of treatment can produces adverse effects through non-specific immunological activation, which may lead to the discontinuation of treatment. Therefore, targeting molecular mechanisms underlying the IRAEs is desirable to allow the continuation and completion of treatment. As steroids and immunosuppressive agents reduce $\mathrm{T}$ cell hyperactivation, other molecules could be used to limit the effect of IL-17. Indeed, the increase of this cytokine is significantly associated with the risk of developing severe immune-mediated AEs. Notably, the role of IL-17, as a pro-inflammatory cytokine, has been highlighted in experimental and human autoimmune disorders, including psoriasis, inflammatory bowel disease and multiple sclerosis (65).

A previous study indicated that the active form of vitamin $\mathrm{D}$ is able to exert a preventative effect in experimental models of autoimmune disorders, due partially to its direct suppressive effect on Th17 cells (65). Therefore, since Th17 cells are inhibited by vitamin D in autoimmune disorders, future therapeutic use of vitamin D during treatments with immune checkpoint inhibitors may be helpful in the prevention of IRAEs. Indeed, vitamin D could reinforce the efficacy of combination therapy with anti-CTLA4 and anti-PD1 through the well-known cytotoxic activity (66) and reduction of the incidence of IRAEs, thus avoiding the discontinuation of treatments.

Finally, evidence has demonstrated that the microbiota can regulate the clinical response to cancer therapy and the onset of toxic AEs (67). A previous study has described the association between variations in gut microbiota and the efficacy of immunotherapy with anti-CTLA4 and anti-PD1/PD-L1 checkpoint inhibitors, whose responsiveness is variable among patients (68). In vivo models demonstrated that CTLA4 antagonists induce $\mathrm{T}$ cell-mediated mucosal damage in the duodenum and colon in parallel to the dysregulation of intestinal and fecal microbiota. The inflammatory intestinal microenvironment induces the expansion of Th-17 cells and increases the risk of IRAEs such as colitis. The majority of findings supported that anti-CTLA4 blockade may alter the gut microbiota, thereby enhancing the antitumor activity.

Unlike CTLA4, PD1/PD-L1 blockade does not induce intestinal damage and this may explain the lower incidence of gastro-intestinal IRAEs during this treatment (69). Thus, targeting the microbiota could represent the newest resource to enhance anticancer efficacy and prevent toxicity resulting from immunotherapy.

\section{Acknowledgements}

The present study was partially supported by a grant from the Italian Association for Cancer Research (grant no. IG11647; Franco Silvestris) and from the Apulia Region Oncogenomic Project (grant no. 2582, 30-12-2013). The authors would also like to thank Associazione per la Ricerca Biomolecolare Onlus for supporting the publication of this paper.

\section{References}

1. Yang Y: Cancer immunotherapy: Harnessing the immune system to battle cancer. J Clin Invest 125: 3335-3337, 2015.

2. Vinay DS, Ryan EP, Pawelec G, Talib WH, Stagg J, Elkord E, Lichtor T, Decker WK, Whelan RL, Kumara HM, et al: Immune evasion in cancer: Mechanistic basis and therapeutic strategies. Semin Cancer Biol 35 (Suppl): S185-S198, 2015.

3. Gajewski TF, Schreiber H and Fu YX: Innate and adaptive immune cells in the tumor microenvironment. Nat Immunol 14: 1014-1022, 2013

4. Pardoll DM: The blockade of immune checkpoints in cancer immunotherapy. Nat Rev Cancer 12: 252-264, 2012.

5. Callahan MK, Postow MA and Wolchok JD: CTLA-4 and PD-1 pathway blockade: Combinations in the clinic. Front Oncol 4: $385,2015$.

6. Tucci M, Stucci S, Passarelli A, Giudice G, Dammacco F and Silvestris F: The immune escape in melanoma: Role of the impaired dendritic cell function. Expert Rev Clin Immunol 10: 1395-1404, 2014

7. Hodi FS, O'Day SJ, McDermott DF, Weber RW, Sosman JA, Haanen JB, Gonzalez R, Robert C, Schadendorf D, Hassel JC, et al: Improved survival with ipilimumab in patients with metastatic melanoma. N Engl J Med 363: 711-723, 2010.

8. Swaika A, Hammond WA and Joseph RW: Current state of anti-PD-L1 and anti-PD-1 agents in cancer therapy. Mol Immunol 67: 4-17, 2015.

9. Wolchok JD, Kluger H, Callahan MK, Postow MA, Rizvi NA, Lesokhin AM, Segal NH, Ariyan CE, Gordon RA, Reed K, et al: Nivolumab plus ipilimumab in advanced melanoma. N Engl J Med 369: 122-133, 2013.

10. Tarhini A: Immune-mediated adverse events associated with ipilimumab ctla-4 blockade therapy: The underlying mechanisms and clinical management. Scientifica (Cairo) 2013: 857519, 2013.

11. Michot JM, Bigenwald C, Champiat S, Collins M, Carbonnel F, Postel-Vinay S, Berdelou A, Varga A, Bahleda R, Hollebecque A, et al: Immune-related adverse events with immune checkpoint blockade: A comprehensive review. Eur J Cancer 54: 139-148, 2016.

12. Larkin J, Chiarion-Sileni V, Gonzalez R, Grob JJ, Cowey CL, Lao CD, Schadendorf D, Dummer R, Smylie M, Rutkowski P, et al: Combined nivolumab and ipilimumab or monotherapy in untreated melanoma. N Engl J Med 373: 23-34, 2015. 
13. Hodi FS, Chesney J, Pavlick AC, Robert C, Grossmann KF, McDermott DF, Linette GP, Meyer N, Giguere JK, Agarwala SS, et al: Combined nivolumab and ipilimumab versus ipilimumab alone in patients with advanced melanoma: 2-year overall survival outcomes in a multicentre, randomised, controlled, phase 2 trial. Lancet Oncol 17: 1558-1568, 2016.

14. Topalian SL, Hodi FS, Brahmer JR, Gettinger SN, Smith DC, McDermott DF, Powderly JD, Carvajal RD, Sosman JA, Atkins MB, et al: Safety, activity, and immune correlates of anti-PD-1 antibody in cancer. N Engl J Med 366: 2443-2454, 2012.

15. Devaud C, John LB, Westwood JA, Darcy PK and Kershaw MH: Immune modulation of the tumor microenvironment for enhancing cancer immunotherapy. Oncoimmunology 2: e25961, 2013.

16. Topalian SL, Sznol M, McDermott DF, Kluger HM, Carvajal RD, Sharfman WH, Brahmer JR, Lawrence DP, Atkins MB, Powderly JD, et al: Survival, durable tumor remission, and long-term safety in patients with advanced melanoma receiving nivolumab. J Clin Oncol 32: 1020-1030, 2014.

17. Weber JS, Dummer R, de Pril V, Lebbé C and Hodi FS MDX010-20 Investigators: Patterns of onset and resolution of immune-related adverse events of special interest with ipilimumab: Detailed safety analysis from a phase 3 trial in patients with advanced melanoma. Cancer 119: 1675-1682, 2013

18. Wolchok JD, Neyns B, Linette G, Negrier S, Lutzky J, Thomas L, Waterfield W, Schadendorf D, Smylie M, Guthrie T Jr, et al: Ipilimumab monotherapy in patients with pretreated advanced melanoma: A randomised, double-blind, multicentre, phase 2 , dose-ranging study. Lancet Oncol 11: 155-164, 2010.

19. Abdel-Rahman O, ElHalawani $\mathrm{H}$ and Fouad M: Risk of gastrointestinal complications in cancer patients treated with immune checkpoint inhibitors: A meta-analysis. Immunotherapy 7 : 1213-1227, 2015.

20. Postow MA, Callahan MK and Wolchok JD: Immune checkpoint blockade in cancer therapy. J Clin Oncol 33: 1974-1982, 2015.

21. Robert C, Schachter J, Long GV, Arance A, Grob JJ, Mortier L, Daud A, Carlino MS, McNeil C, Lotem M, et al: Pembrolizumab versus ipilimumab in advanced melanoma. N Engl J Med 372: 2521-2532, 2015

22. Bertrand A, Kostine M, Barnetche T, Truchetet ME and Schaeverbeke T: Immune related adverse events associated with anti-CTLA-4 antibodies: Systematic review and meta-analysis. BMC Med 13: 211, 2015.

23. Beck KE, Blansfield JA, Tran KQ, Feldman AL, Hughes MS Royal RE, Kammula US, Topalian SL, Sherry RM, Kleiner D, et al: Enterocolitis in patients with cancer after antibody blockade of cytotoxic T-lymphocyte-associated antigen 4 J Clin Oncol 24: 2283-2289, 2006

24. Ibrahim RA, Berman DM, DePril V, Humphrey RW, Chen T, Messina M, Chin KM, Liu HY, Bielefield M and Hoos A: Ipilimumab safety profile: Summary of findings from completed trials in advanced melanoma. J Clin Oncol 29, 2011.

25. Robert C, Ribas A, Wolchok JD, Hodi FS, Hamid O, Kefford R, Weber JS, Joshua AM, Hwu WJ, Gangadhar TC, et al: Anti-p rogrammed-death-receptor-1 treatment with pembrolizumab in ipilimumab-refractory advanced melanoma: A randomised dose-comparison cohort of a phase 1 trial. Lancet 384: 1109-1117, 2014.

26. Zimmer L, Goldinger SM, Hofmann L, Loquai C Ugurel S, Thomas I, Schmidgen MI, Gutzmer R, Utikal JS, Göppner D, et al: Neurological, respiratory, musculoskeletal, cardiac and ocular side-effects of anti-PD-1 therapy. Eur J Cancer 60: 210-225, 2016.

27. Faje A: Immunotherapy and hypophysitis: Clinical presentation, treatment and biologic insights. Pituitary 19: 82-92, 2016.

28. Hofmann L, Forschner A, Loquai C, Goldinger SM, Zimmer L, Ugurel S, Schmidgen MI, Gutzmer R, Utikal JS, Göppner D, et al: Cutaneous, gastrointestinal, hepatic, endocrine and renal side-effects of anti-PD-1 therapy. Eur J Cancer 60: 190-209, 2016

29. Barjaktarevic IZ, Qadir N, Suri A, Santamauro JT and Stover D Organizing pneumonia as a side effect of ipilimumab treatment of melanoma. Chest 143: 858-861, 2013.

30. Abdel-Rahman $\mathrm{O}$ and Fouad M: Risk of pneumonitis in cancer patients treated with immune checkpoint inhibitors: A meta-analysis. Ther Adv Respir Dis 10: 183-193, 2016.

31. Papavasileiou E, Prasad S, Freitag SK, Sobrin L and Lobo AM: Ipilimumab-induced ocular and orbital inflammation-A case series and review of the literature. Ocul Immunol Inflamm 24: $140-146,2016$
32. Bot I, Blank CU, Boogerd W and Brandsma D: Neurological immune-related adverse events of ipilimumab. Pract Neurol 13: 278-280, 2013.

33. Cappelli LC, Shah AA and Bingham CO III: Immune-related adverse effects of cancer immunotherapy-implications for rheumatology. Rheum Dis Clin North Am 43: 65-78, 2017.

34. Goldstein BL, Gedmintas L and Todd DJ: Drug-associated polymyalgia rheumatica/giant cell arteritis occurring in two patients after treatment with ipilimumab, an antagonist of ctla-4. Arthritis Rheumatol 66: 768-769, 2014.

35. Fadel F, El Karoui K and Knebelmann B: Anti-CTLA4 antibody-induced lupus nephritis. N Engl J Med 361: 211-212, 2009.

36. Forde PM,Rock K,Wilson Gand O'ByrneKJ: Ipilimumab-induced immune-related renal failure--a case report. Anticancer Res 32: 4607-4608, 2012

37. Gordon IO, Wade T, Chin K, Dickstein J and Gajewski TF: Immune-mediated red cell aplasia after anti-CTLA-4 immunotherapy for metastatic melanoma. Cancer Immunol Immunother 58: 1351-1353, 2009.

38. Guo Y and Wang AY: Novel immune check-point regulators in tolerance maintenance. Fron Immunol 6: 421, 2015.

39. Ueda H, Howson JM, Esposito L, Heward J, Snook H, Chamberlain G, Rainbow DB, Hunter KM, Smith AN, Di Genova G, et al: Association of the T-cell regulatory gene CTLA4 with susceptibility to autoimmune disease. Nature 423 : 506-511, 2003.

40. Jin P, Xiang B, Huang $\mathrm{G}$ and Zhou Z: The association of cytotoxic T-lymphocyte antigen $-4+49 \mathrm{~A} / \mathrm{G}$ and CT60 polymorphisms with type 1 diabetes and latent autoimmune diabetes in Chinese adults. J Endocrinol Invest 38: 149-154, 2015.

41. Kaehler KC, Piel S, Livingstone E, Schilling B, Hauschild A and Schadendorf D: Update on immunologic therapy with anti-CTLA-4 antibodies in melanoma: Identification of clinical and biological response patterns, immune-related adverse events, and their management. Semin Oncol 37: 485-498, 2010.

42. Berman D, Parker SM, Siegel J, Chasalow SD, Weber J, Galbraith S, Targan SR and Wang HL: Blockade of cytotoxic T-lymphocyte antigen-4 by ipilimumab results in dysregulation of gastrointestinal immunity in patients with advanced melanoma. Cancer Immun 10: 11, 2010.

43. Nancey S, Boschetti G, Cotte E, Ruel K, Almeras T, Chauvenet M, Stroeymeyt K, Moussata D, Kaiserlian D and Flourié B: Blockade of cytotoxic T-lymphocyte antigen-4 by ipilimumab is associated with a profound long-lasting depletion of Foxp3+ regulatory T cells: A mechanistic explanation for ipilimumab-induced severe enterocolitis? Inflamm Bowel Dis 18: E1598-E1600, 2012.

44. Lord JD, Hackman RC, Moklebust A, Thompson JA, Higano CS, Chielens D, Steinbach G and McDonald GB: Refractory colitis following anti-CTLA4 antibody therapy: Analysis of mucosal FOXP3+ T cells. Dig Dis Sci 55: 1396-1405, 2010

45. Callahan MK, Yang A, Tandon S, Xu Y, Subudhi SK, Roman RA, Heine AI, Pogoriler E, Kuk D, Panageas K, et al: Evaluation of serum IL-17 levels during ipilimumab therapy: Correlation with colitis. J Clin Oncol 29, 2011.

46. Kähler KC and Hauschild A: Treatment and side effect management of CTLA-4 antibody therapy in metastatic melanoma. J Dtsch Dermatol Ges 9: 277-286, 2011 (In English, German)

47. Lacouture ME, Wolchok JD, Yosipovitch G, Kähler KC, Busam KJ and Hauschild A: Ipilimumab in patients with cancer and the management of dermatologic adverse events. J Am Acad Dermatol 71: 161-169, 2014

48. Weber J, Thompson JA, Hamid O, Minor D, Amin A, Ron I, Ridolfi R, Assi H, Maraveyas A, Berman D, et al: A randomized, double-blind, placebo-controlled, phase II study comparing the tolerability and efficacy of ipilimumab administered with or without prophylactic budesonide in patients with unresectable stage III or IV melanoma. Clin Cancer Res 15: 5591-5598, 2009.

49. Weber JS, Kähler KC and Hauschild A: Management of immune-related adverse events and kinetics of response with ipilimumab. J Clin Oncol 30: 2691-2697, 2012

50. Villadolid $\mathrm{J}$ and Amin A: Immune checkpoint inhibitors in clinical practice: Update on management of immune-related toxicities. Transl Lung Cancer Res 4: 560-575, 2015.

51. Weber JS, Berman D, Siegel J, Minor D, Amin A, Thompson JA, Ron I, Ridolfi R, Assi H and Hamid O: Safety and efficacy of ipilimumab with or without prophylactic budesonide in treatment-naive and previously treated patients with advanced melanoma. J Clin Oncol, 2008

52. Spain L, Diem S and Larkin J: Management of toxicities of immune checkpoint inhibitors. Cancer Treat Rev 44: 51-60, 2016. 
53. Friedman CF, Proverbs-Singh TA and Postow MA: Treatment of the immune-related adverse effects of immune checkpoint inhibitors: A review. JAMA Oncol 2: 1346-1353, 2016.

54. Sanlorenzo M, Vujic I, Daud A, Algazi A, Gubens M, Luna SA, Lin K, Quaglino P, Rappersberger K and Ortiz-Urda S: Pembrolizumab cutaneous adverse events and their association with disease progression. JAMA Dermatol 151: 1206-1212, 2015.

55. Lacouture ME: Management of dermatologic toxicities. J Natl Compr Canc Netw 13 (5 Suppl): S686-S689, 2015.

56. Khunger M, Rakshit S, Pasupuleti V, Hernandez AV, Mazzone P, Stevenson J, Pennell NA and Velcheti V: Incidence of pneumonitis with use of PD-1 and PD-L1 inhibitors in non-small cel lung cancer: A systematic review and meta-analysis of trials. Chest 152: 271-281, 2017

57. Tirumani SH, Ramaiya NH, Keraliya A, Bailey ND, Ott PA Hodi FS and Nishino M: Radiographic profiling of immune-related adverse events in advanced melanoma patients treated with ipilimumab. Cancer Immunol Res 3: 1185-1192, 2015.

58. Nishino M, Chambers ES, Chong CR, Ramaiya NH, Gray SW, Marcoux JP, Hatabu H, Jänne PA, Hodi FS and Awad MM Anti-PD-1 inhibitor-related pneumonitis in non-small cell lung cancer. Cancer Immunol Res 4: 289-293, 2016.

59. Dillard T, Yedinak CG, Alumkal J and Fleseriu M: Anti-CTLA-4 antibody therapy associated autoimmune hypophysitis: Serious immune related adverse events across a spectrum of cancer subtypes. Pituitary 13: 29-38, 2010.

60. Lammert A, Schneider HJ, Bergmann T, Benck U, Krämer BK, Gärtner R, Metzner C, Schöfl C and Berking C: Hypophysitis caused by ipilimumab in cancer patients: Hormone replacement or immunosuppressive therapy. Exp Clin Endocrinol Diabetes 121: 581-587, 2013.

61. González-Rodríguez E and Rodríguez-Abreu D; Spanish Group for Cancer Immuno-Biotherapy (GETICA): Immune checkpoint inhibitors: Review and management of endocrine adverse events. Oncologist 21: 804-816, 2016.
62. Orlov S, Salari F, Kashat L and Walfish PG: Induction of painless thyroiditis in patients receiving programmed death 1 receptor immunotherapy for metastatic malignancies. J Clin Endocrinol Metab 100: 1738-1741, 2015.

63. Amin A, DePril V, Hamid O, Wolchock J, Maio M, Neyns B, Chin K, Ibrahim R, Hoos A and O'Day S: Evaluation of the effect of systemic corticosteroids for the treatment of immune-related adverse events (irAEs) on the development or maintenance of ipilimumab clinical activity. J Clin Oncol 27, 2009.

64. Suarez-Almazor ME, Kim ST, Abdel-Wahab N and Diab A Review: Immune-related adverse events with use of checkpoint inhibitors for immunotherapy of cancer. Arthritis Rheumatol 69: 687-699, 2017.

65. Joshi S, Pantalena LC, Liu XK, Gaffen SL, Liu H, Rohowsky-Kochan C, Ichiyama K, Yoshimura A, Steinman L, Christakos S and Youssef S: 1,25-dihydroxyvitamin D (3) ameliorates Th17 autoimmunity via transcriptional modulation of interleukin-17A. Mol Cell Biol 31: 3653-3669, 2011.

66. Deeb KK, Trump DL and Johnson CS: Vitamin D signalling pathways in cancer: Potential for anticancer therapeutics. Nat Rev Cancer 7: 684-700, 2007.

67. Roy S and Trinchieri G: Microbiota: A key orchestrator of cancer therapy. Nat Rev Cancer 17: 271-285, 2017.

68. Vétizou M, Pitt JM, Daillère R, Lepage $P$, Waldschmitt $N$, Flament C, Rusakiewicz S, Routy B, Roberti MP, Duong CP, et al: Anticancer immunotherapy by CTLA-4 blockade relies on the gut microbiota. Science 350: 1079-1084, 2015.

69. Sivan A, Corrales L, Hubert N, Williams JB, Aquino-Michaels K, Earley ZM, Benyamin FW, Lei YM, Jabri B, Alegre ML, et al: Commensal Bifidobacterium promotes antitumor immunity and facilitates anti-PD-L1 efficacy. Science 350: 1084-1089, 2015. 